\title{
Neuronal Dysfunction and Medical Therapy in Heart Failure: Can an Imaging Biomarker Help to "Personalize" Therapy?
}

\author{
Benjamin S. Wessler ${ }^{1,2}$ and James E. Udelson ${ }^{1}$ \\ ${ }^{I}$ Division of Cardiology and CardioVascular Center, Tufts Medical Center, Tufts University School of Medicine, Boston \\ Massachusetts; and ${ }^{2}$ Predictive Analytics and Comparative Effectiveness (PACE) Center, Tufts Medical Center, Boston, \\ Massachusetts
}

\begin{abstract}
${ }^{123}$ I-metaiodobenzylguanidine (123I-MIBG) imaging is a tool for evaluating one of the fundamental pathophysiologic abnormalities seen in heart failure (HF), that of an upregulated sympathetic nervous system and its effect on the myocardium. Although this imaging technique offers information about prognosis for patients treated with contemporary guideline-based HF therapies and improves risk stratification, there are neither rigorous nor sufficient outcome data to suggest that this imaging tool can guide therapeutic decision making or better target subsets of patients with HF for particular therapies.
\end{abstract}

Key Words: heart failure; heart-to-mediastinum ratio; myocardial washout rate; norepinephrine

J Nucl Med 2015; 56:20S-24S

DOI: 10.2967/jnumed.114.142778

$\mathbf{T}$

here have been substantial advances in therapeutics over the past 3 decades for patients with heart failure (HF), with resulting extensive evidence-based recommendations for treatment (1). Although hemodynamic changes dominate the daily clinical syndrome, neurohormonal alterations drive the pathophysiologic progression of HF associated with longer-term outcomes and are the major targets for contemporary drug therapy $(1,2)$. Upregulation of norepinephrine (NE) is associated with a poor prognosis for patients with chronic HF (3). Imaging techniques are now available to evaluate adaptations to the cardiac sympathetic nervous system and thus have the potential to more directly interrogate an important pathophysiologic feature of chronic HF with systolic dysfunction $(3,4)$.

For patients with HF, myocardial synaptic physiology involving NE can be studied by labeling the NE analog guanethidine with radiolabeled iodine. The resulting compound, ${ }^{123}$ I-metaiodobenzylguanidine ( $\left.{ }^{123} \mathrm{I}-\mathrm{MIBG}\right)$, acts like NE with respect to movement into and out of the synapse. Because it is not catabolized like NE, it is retained within myocardial nerve endings and can be imaged (5). Various measures of ${ }^{123}$ I-MIBG uptake, such as the heart-tomediastinum ratio (HMR) and myocardial washout rate, allow investigators to characterize myocardial uptake and thus obtain

Received Dec. 02, 2014; revision accepted Jan. 14, 2015.

For correspondence or reprints contact: James E. Udelson, Box 70 Cardiology, Tufts Medical Center, 800 Washington St., Boston MA 02111.

E-mail: JUdelson@tuftsmedicalcenter.org

COPYRIGHT (C 2015 by the Society of Nuclear Medicine and Molecular Imaging, Inc. a noninvasive assessment of cardiac NE receptor density and functional sympathetic innervation $(6,7)$. In general, individuals with healthy hearts have high cardiac uptake of ${ }^{123}$ I-MIBG, whereas those with HF have lower myocardial uptake, reflecting decreased cardiac adrenergic receptor density.

Seminal work done by Merlet et al. demonstrated the value of ${ }^{123}$ I-MIBG imaging as a prognostic tool for patients with HF (8). These investigators evaluated ${ }^{123}$ I-MIBG uptake in 90 patients with $\mathrm{HF}$, a left ventricular ejection fraction (LVEF) of less than $45 \%$, and New York Heart Association (NYHA) class II-IV symptoms and showed that the ${ }^{123}$ I-MIBG HMR was associated with overall survival during follow-up, which ranged from 1 to 27 mo. During the 20-plus years that have passed since this original observation, treatment and outcomes for patients with HF have improved (9). In response to changing care patterns, there has been great interest in understanding whether the observations of Merlet et al. (8) apply to patients treated with current guideline-based HF therapies.

The AdreView Myocardial Imaging for Risk Evaluation in Heart Failure (ADMIRE-HF) trial addressed this topic by prospectively monitoring 961 subjects with HF at 96 centers in the United States, Canada, and Europe after ${ }^{123}$ I-MIBG imaging (10). Patients had NYHA class II or III symptoms, had an LVEF of less than or equal to $35 \%$, were being treated with guideline-based HF therapies, and were monitored for up to $2 \mathrm{y}$. The results suggested that the observations of Merlet et al. (8) from 1992 appear to be reproducible in the setting of modern HF therapies. In the ADMIRE-HF trial, the risk of cardiac events (a composite of time to cardiac death, life-threatening arrhythmic event, or NYHA functional class progression) was significantly lower for participants with an HMR of greater than or equal to 1.6 than for those with an HMR of less than 1.6 (hazard ratio, $0.40 ; 97.5 \%$ confidence interval, $0.25-0.64 ; P<0.001)$. Although the findings from the ADMIRE$\mathrm{HF}$ trial support the predictive value of this imaging technique for assessing general prognosis, the ADMIRE-HF trial program was not designed to show that clinical decisions can be influenced by the results of ${ }^{123}$ I-MIBG imaging. Indeed, regulatory authorities recognized this limitation, as the prescribing information for this agent states that its “. . utility has not been established for selecting a therapeutic intervention or for monitoring the response to therapy" (11).

Numerous investigators have examined the interplay of HF therapies and ${ }^{123}$ I-MIBG assessment of the integrity of sympathetic innervation. These studies generally have taken 1 of 2 analytic approaches. One approach investigates the effect of a therapy, such as angiotensin-converting enzyme (ACE) inhibition, on measures of ${ }^{123}$ I-MIBG uptake evaluated before treatment and 
again at a later time (during treatment). This approach attempts to study pathophysiology of the treatment effect, that is, how a treatment (which is known to be clinically effective) affects sympathetic innervation. Many such studies are summarized in Table 1. The other approach involves examining the association between baseline ${ }^{123}$ I-MIBG data obtained before treatment and some response variable (such as a change in LVEF) resulting from the treatment. This analytic methodology attempts to use ${ }^{123} \mathrm{I}-$ MIBG data to predict which patients might have a "better" response to a treatment. Studies in which this approach was used are summarized in Table 2.

The studies summarized in Table 1 showed that therapies known to be clinically effective in patients with HF, predominantly neurohormonal antagonists, seemed to be consistently associated with improvement in measures of ${ }^{123}$ I-MIBG uptake or washout. These data, in turn, suggested that such therapies are associated with improvement in functional sympathetic innervation. Whether this change in an underlying physiologic substrate is part of the causal pathway of the clinically favorable effects of these therapies cannot be determined from such studies. It is conceivable that the observed change in sympathetic innervation is secondary to other, more fundamental effects of the pharmacologic therapies, such as improvement in left ventricular size or function or effects on baroreceptors. Although these studies are of significant interest from a physiologic point of view and raise interesting questions, they do not by themselves illuminate treatment pathways or mechanisms.

Published reviews of ${ }^{123}$ I-MIBG imaging in HF patients often raise the possibility that ${ }^{123}$ I-MIBG imaging may be useful in "...judging the likely result of medical or device therapy..." (4). The studies detailed in Table 1 did not address this issue. The idea that an imaging biomarker might be used to "select" patients who may have a greater or a lesser response to a therapy-thereby allowing consideration of the use or nonuse of the therapy-can be potentially informed by studies such as those summarized in Table 2, in which some aspect of baseline ${ }^{123}$ I-MIBG data

TABLE 1

Effects of HF Therapies on Measures of ${ }^{123}$ I-MIBG Uptake and Washout

\begin{tabular}{|c|c|c|c|c|c|}
\hline Study & Therapy & No. of patients & Follow-up & Design & Results \\
\hline Barr et al. (28) & Spironolactone/placebo & 42 & $8 w k$ & RCT & $\begin{array}{l}\text { Improved HMR in } \\
\text { spironolactone group }\end{array}$ \\
\hline Somsen et al. (29) & Enalapril & 23 & $6 \mathrm{wk}$ & Pre/post uncontrolled & $\begin{array}{l}\text { Improved SPECT } \\
\text { uptake after enalapril }\end{array}$ \\
\hline Fukuoka et al. (30) & Metoprolol & 13 & $3 \mathrm{mo}$ & $\begin{array}{l}\text { Nonrandomized, } \\
\text { uncontrolled }\end{array}$ & $\begin{array}{l}\text { Patients with change in } \\
E F \geq 5 \% \text { had more } \\
\text { improvement in WOR }\end{array}$ \\
\hline Takeishi et al. (31) & Enalapril/control & 29 & $9 \mathrm{mo}$ & Nonrandomized & $\begin{array}{l}\text { Improved HMR and } \\
\text { WOR in enalapril } \\
\text { group }\end{array}$ \\
\hline Soeki et al. (32) & Enalapril & 10 & $7 \mathrm{mo}$ & Pre/post uncontrolled & $\begin{array}{l}\text { Improved HMR but not } \\
\text { WOR in enalapril } \\
\text { group }\end{array}$ \\
\hline Toyama et al. (33) & Metoprolol/enalapril & 24 & $12 \mathrm{mo}$ & $\begin{array}{l}\text { Randomized active } \\
\text { control }\end{array}$ & $\begin{array}{l}\text { Improved HMR in } \\
\text { enalapril and } \\
\text { metoprolol groups }\end{array}$ \\
\hline Agostini et al. (34) & Carvedilol & 22 & $6 \mathrm{mo}$ & Pre/post uncontrolled & $\begin{array}{l}\text { Improved HMR after } \\
\text { carvedilol }\end{array}$ \\
\hline Gerson et al. (18) & Carvedilol & 22 & $7 \mathrm{mo}$ & Pre/post uncontrolled & $\begin{array}{l}\text { Improved HMR after } \\
\text { carvedilol }\end{array}$ \\
\hline Kasama et al. (35) & Spirinolactone & 30 & $6 \mathrm{mo}$ & $\mathrm{RCT}$ & $\begin{array}{l}\text { Improved HMR and } \\
\text { WOR in } \\
\text { spironolactone group }\end{array}$ \\
\hline Toyama et al. (36) & Amiodarone/metoprolol & 30 & $12 \mathrm{mo}$ & $\begin{array}{l}\text { Randomized active } \\
\text { control }\end{array}$ & $\begin{array}{l}\text { Improved HMR in } \\
\text { amiodarone and } \\
\text { metoprolol groups }\end{array}$ \\
\hline Kasama et al. (37) & Candesartan/placebo & 50 & $6 \mathrm{mo}$ & $\begin{array}{l}\text { RCT (in patients with } \\
\text { HFpEF) }\end{array}$ & $\begin{array}{l}\text { Improved HMR and } \\
\text { WOR in candesartan } \\
\text { group }\end{array}$ \\
\hline
\end{tabular}

Pre/post = before and after drug treatment; RCT = randomized controlled trial; WOR = washout rate for ${ }^{123} \mid-\mathrm{MIBG}$; HFpEF $=$ heart failure with preserved ejection fraction. 
TABLE 2

123/-MIBG Uptake or Washout at Baseline as Predictor of Differential Treatment Effect

\begin{tabular}{|c|c|c|c|c|c|}
\hline Study & Therapy & No. of patients & Follow-up & Design & Results \\
\hline Suwa et al. (38) & Bisoprolol & 45 & $8 \mathrm{mo}$ & Uncontrolled & $\begin{array}{l}\text { Baseline HMR associated with } \\
\text { "response," defined as both } \\
\text { reverse remodeling and } \\
\text { improvement in NYHA class }\end{array}$ \\
\hline Choi et al. (15) & Carvedilol & 11 & $12 \mathrm{mo}$ & $\mathrm{RCT}$ & $\begin{array}{l}\text { Inverse correlation between } \\
\text { baseline WOR and change } \\
\text { in LVEF }\end{array}$ \\
\hline Gerson et al. (18) & Carvedilol & 22 & $7 \mathrm{mo}$ & Uncontrolled & $\begin{array}{l}\text { No relationship between } \\
\text { baseline HMR and change } \\
\text { in LVEF }\end{array}$ \\
\hline Yamazaki et al. (16) & $\beta$-blockers & 40 & $6 \mathrm{mo}$ & Uncontrolled & $\begin{array}{l}\text { Lower mean extent and } \\
\text { severity uptake scores at } \\
\text { baseline in patients with } \\
\text { change in LVEF of }>10 \%\end{array}$ \\
\hline
\end{tabular}

obtained before treatment is correlated with a measure of response to the treatment. The studies shown in Table 2 are not the result of a formal survey of the literature, as in a formal systematic review; rather, these studies were compiled from an examination of several reviews that have been published in the past $15 \mathrm{y}(4,6,12)$.

Several insights can be gleaned from the studies shown in Table 2. First, there are very few such studies, and they incorporated very few patients. Second, none evaluated true patient-related clinical outcomes. They evaluated surrogates, such as change in ejection fraction or remodeling, that are related to outcomes in a general way $(13,14)$ but are not highly predictive. Third, the data do not resonate with the results of seminal clinical trials. In the study of Choi et al. (15) (Table 2), there was an inverse correlation between the baseline ${ }^{123}$ I-MIBG washout rate and a change in LVEF over 12 mo of carvedilol therapy; in other words, the higher the washout rate (more abnormal), the lower the change in the LVEF in response to carvedilol. In the study of Yamakazi et al. (16), patients with HF and the least abnormal ${ }^{123}$ I-MIBG uptake had a better LVEF response to $\beta$-blockers. Hence, the patients with the most abnormal ${ }^{123}$ I-MIBG profiles-who were generally the patients with the most advanced $\mathrm{HF}$ - had the least favorable LVEF responses in these 2 studies. However, in the COPERNICUS trial (17), involving 2,289 patients with severe HF symptoms (NYHA class IV) and an LVEF of less than 30\%, carvedilol had a very favorable clinical effect. In fact, the effect of carvedilol on survival was so impactful that the COPERNICUS trial was halted early, as the survival benefit exceeded the prespecified monitoring boundaries on an interim analysis. Thus, in rigorously executed clinical trials, patients with very advanced symptoms and left ventricular dysfunction had a very favorable outcome response to carvedilol, inconsistent with the "prediction" of the ${ }^{123}$ I-MIBG studies.

Finally, the data from the small number of studies shown in Table 2 were not internally consistent. Although 3 of the studies suggested that some baseline measure of ${ }^{123}$ I-MIBG correlates with a treatment effect of $\beta$-blockers on the LVEF, Gerson et al. (18) reported no such relationship. The latter study is actually more consistent with the large clinical trial literature, as none of the major $\beta$-blocker randomized trials, involving thousands of patients, has identified a clinically relevant subgroup of patients in whom there is a clear differential effect on outcomes; that is, there is no subgroup of patients for whom treatment with $\beta$-blockers is not attempted. Thus, the few ${ }^{123} \mathrm{I}-\mathrm{MIBG}$ studies shown in Table 2 provide no indication that imaging with ${ }^{123} \mathrm{I}-\mathrm{MIBG}$ might yield clinical information regarding drug selection for patients with HF.

It has also been suggested that ${ }^{123}$ I-MIBG imaging may assist in the selection of patients for implantable cardioverter defibrillators (ICDs) to better target this expensive therapy. Although it is biologically plausible that a measure of preserved sympathetic innervation might identify a subgroup with such a very low risk for arrhythmic events that an ICD may not be needed, at this point the actual data supporting such a concept are not strong. In the first study to address this issue, Arora et al. (19) reported on a group of 17 patients who had ICDs and were evaluated with ${ }^{123}$ I-MIBG imaging and heart rate variability analysis. A group of patients who had no ICD discharges (and who, in theory, would not benefit from ICDs) was characterized by both preserved ${ }^{123}$ I-MIBG uptake and preserved (more normal) heart rate variability, but this group consisted of only 3 patients. In a recent analysis of the ADMIRE-HF trial program, Al Badarin et al. (20) created a risk score for arrhythmic events (the events that would be most directly affected by an ICD) and identified a "low-risk" group of 153 patients in whom only 3 events occurred, for a crude event rate estimate of $2 \%$. The authors acknowledged the absence of external validation but concluded that the data suggested that ${ }^{123} \mathrm{I}-\mathrm{MIBG}$ imaging in such patients could have a "... role in individualizing arrhythmic risk assessment and improving decisions for device implantation," by which they presumably meant identifying a low-risk group of patients who may not benefit from an ICD. As pointed out in an accompanying editorial (21), even a risk of $2 \%$ may not be low enough to rule out the potential benefit of an ICD. Moreover, authors (such as Al Badarin et al. (20)) often focus on point estimates of risk in a studied population. More important is to examine the confidence intervals around the point estimate, as the true risk in the studied population-if it could ever 
be measured-might be distinctly higher than $2 \%$, especially given the very small number of events (3) in the small population (153 patients) studied. An estimation of the upper bound of the 95\% confidence interval from the data in the study of $\mathrm{Al}$ Baradin et al. (20) puts the possible upper bound of risk in this "low-risk" group of patients as high as $4.2 \%$, which is not "low."

The data and level of evidence required for a serum or imaging biomarker to enter clinical use in order to not treat selected patients with an existing guideline-based therapy is very high. Are there any examples in the therapeutic realm of patients with HF? One such example is the evolution of recommendations for cardiac resynchronization therapy (CRT) and the influence of an imaging "biomarker," the electrocardiogram. As recently as 2009, guidelines for HF recommended CRT as a class I indication for patients with symptomatic HF, an LVEF of less than or equal to $35 \%$, and a QRS duration of greater than or equal to $120 \mathrm{~ms}$ (22). In the 2013 guidelines, however, the class I CRT indication more specifically (and narrowly) states that patients should have a left bundle branch block configuration as the driver of the wide QRS (1). In other words, a right bundle branch block or non-left bundle branch block driver of a wide QRS is no longer a strong indication for CRT. The data that influenced the change in the recommendation were very strong; they ranged from consistent subgroup analyses of individual large randomized controlled CRT outcome trials $(23,24)$ to metaanalyses of major randomized trials involving more than 5,000 patients and Medicare registry data involving almost 15,000 patients, all with actual clinical outcomes $(25,26)$, not surrogates. The evidence for the use of ${ }^{123}$ I-MIBG imaging to "narrow" or "target" drug or device therapy is scant relative to the data for CRT.

Since the seminal study of Merlet et al. (8), however, the published data are quite consistent regarding the association of ${ }^{123}$ IMIBG imaging results with future risk of various events in patients with HF. Using contemporary analytic approaches to evaluate biomarkers for prognosis, investigators assessed whether ${ }^{123} \mathrm{I}$ MIBG uptake can improve currently available risk stratification tools. Ketchum et al. determined the incremental value of adding ${ }^{123}$ I-MIBG uptake data to the Seattle Heart Failure Model (27). Using patient-level data from the ADMIRE-HF trial, they found that the addition of the ${ }^{123}$ I-MIBG HMR to the calculated Seattle Heart Failure Model score improved risk stratification (the net reclassification improvement was 22.7\%; $P<0.001$ ). As with most risk stratification studies, however, the clinical value of this observation is unclear because no therapeutic decision is informed by this new information. Ever finer gradations of statistically significant risk stratification or reclassification do not clearly result in any differential clinical decision for most patients with HF, whether the marker is a serum biomarker or an imaging biomarker.

${ }^{123}$ I-MIBG imaging is a tool for evaluating one of the fundamental pathophysiologic abnormalities seen in HF, that of an upregulated sympathetic nervous system and its effect on the myocardium. Although this imaging technique offers information about prognosis for patients treated with contemporary guideline-based HF therapies and improves risk stratification, there are neither rigorous nor sufficient outcome data to suggest that this imaging technique can guide therapeutic decision making or better target subsets of patients with HF for particular therapies. The lessons from the evolution of CRT indications suggest that it is possible to better target therapies; however, large studies with designs and analyses focused on this topic and appropriate patient populations are essential, and they do not currently exist for ${ }^{123}$ I-MIBG. Until such a trial or trial program can be conceived and executed and ${ }^{123} \mathrm{I}-\mathrm{MIBG}$ imaging can be shown to identify a new subgroup of patients who have HF and derive (or do not derive) benefit from a given therapy, patients with $\mathrm{HF}$ and a reduced LVEF will continue to be treated with "one-size-fits-all" therapies based on clinical trial summary results and guidelines, and ${ }^{123} \mathrm{I}-\mathrm{MIBG}$ will continue to be a product approved for use but without clear utility.

\section{DISCLOSURE}

Benjamin S. Wessler was supported in part by grant T32HL069770 from the NIH. No other potential conflict of interest relevant to this article was reported.

\section{REFERENCES}

1. Yancy CW, Jessup M, Bozkurt B, et al. 2013 ACCF/AHA guideline for the management of heart failure: a report of the American College of Cardiology Foundation/American Heart Association Task Force on practice guidelines. Circulation. 2013;128:e240-e327.

2. Schrier RW, Abraham WT. Hormones and hemodynamics in heart failure. $N$ Engl J Med. 1999;341:577-585.

3. Cohn JN, Levine TB, Olivari MT, et al. Plasma norepinephrine as a guide to prognosis in patients with chronic congestive heart failure. $N$ Engl J Med. 1984;311:819-823.

4. Carrió I, Cowie MR, Yamazaki J, Udelson J, Camici PG. Cardiac sympathetic imaging with mIBG in heart failure. JACC Cardiovasc Imaging. 2010;3:92-100.

5. Chirumamilla A, Travin MI. Cardiac applications of ${ }^{123}$ I-mIBG imaging. Semin Nucl Med. 2011;41:374-387.

6. Patel AD, Iskandrian AE. MIBG imaging. J Nucl Cardiol. 2002;9:75-94.

7. Schofer J, Spielmann R, Schuchert A, Weber K, Schlüter M. Iodine-123 metaiodobenzylguanidine scintigraphy: a noninvasive method to demonstrate myocardial adrenergic nervous system disintegrity in patients with idiopathic dilated cardiomyopathy. J Am Coll Cardiol. 1988;12:1252-1258.

8. Merlet P, Valette H, Dubois-Randé JL, et al. Prognostic value of cardiac metaiodobenzylguanidine imaging in patients with heart failure. J Nucl Med. 1992; 33:471-477.

9. Joffe SW, Webster K, McManus DD, et al. Improved survival after heart failure: a community-based perspective. J Am Heart Assoc. 2013;2:e000053.

10. Jacobson AF, Senior R, Cerqueira MD, et al. Myocardial iodine-123 metaiodobenzylguanidine imaging and cardiac events in heart failure: results of the prospective ADMIRE-HF (AdreView Myocardial Imaging for Risk Evaluation in Heart Failure) study. J Am Coll Cardiol. 2010;55:2212-2221.

11. AdreView prescribing information. GE Healthcare website. http://www3. gehealthcare.com/en/products/categories/nuclear_imaging_agents/adreview. Accessed February 23, 2015.

12. Treglia G, Stefanelli A, Bruno I, Giordano A. Clinical usefulness of myocardial innervation imaging using iodine-123-meta-iodobenzylguanidine scintigraphy in evaluating the effectiveness of pharmacological treatments in patients with heart failure: an overview. Eur Rev Med Pharmacol Sci. 2013;17:56-68.

13. Kramer DG, Trikalinos TA, Kent DM, Antonopoulos GV, Konstam MA, Udelson JE. Quantitative evaluation of drug or device effects on ventricular remodeling as predictors of therapeutic effects on mortality in patients with heart failure and reduced ejection fraction: a meta-analytic approach. J Am Coll Cardiol. 2010;56:392-406.

14. Wessler BS, Kramer DG, Kelly JL, et al. Drug and device effects on peak oxygen consumption, 6-minute walk distance, and natriuretic peptides as predictors of therapeutic effects on mortality in patients with heart failure and reduced ejection fraction. Circ Heart Fail. 2011;4:578-588.

15. Choi JY, Lee KH, Hong KP, et al. Iodine-123 MIBG imaging before treatment of heart failure with carvedilol to predict improvement of left ventricular function and exercise capacity. J Nucl Cardiol. 2001;8:4-9.

16. Yamazaki J, Muto H, Kabano T, Yamashina S, Nanjo S, Inoue A. Evaluation of beta-blocker therapy in patients with dilated cardiomyopathy: clinical meaning of iodine 123-metaiodobenzylguanidine myocardial single-photon emission computed tomography. Am Heart J. 2001;141:645-652.

17. Packer M, Coats AJ, Fowler MB, et al. Effect of carvedilol on survival in severe chronic heart failure. N Engl J Med. 2001;344:1651-1658.

18. Gerson MC, Craft LL, McGuire N, Suresh DP, Abraham WT, Wagoner LE. Carvedilol improves left ventricular function in heart failure patients with idiopathic dilated cardiomyopathy and a wide range of sympathetic nervous system 
function as measured by iodine 123 metaiodobenzylguanidine. J Nucl Cardiol. 2002;9:608-615.

19. Arora R, Ferrick KJ, Nakata T, et al. I-123 MIBG imaging and heart rate variability analysis to predict the need for an implantable cardioverter defibrillator. J Nucl Cardiol. 2003;10:121-131.

20. Al Badarin FJ, Wimmer AP, Kennedy KF, Jacobson AF, Bateman TM. The utility of ADMIRE-HF risk score in predicting serious arrhythmic events in heart failure patients: incremental prognostic benefit of cardiac ${ }^{123} \mathrm{I}-\mathrm{mIBG}$ scintigraphy. J Nucl Cardiol. 2014;21:756-762.

21. Saba S, Aban I, Soman P. ${ }^{123}$ I-mIBG scintigraphy: yet another risk stratifier for the heart failure toolbox! J Nucl Cardiol. 2014;21:909-912.

22. Hunt SA, Abraham WT, Chin MH, et al. 2009 focused update incorporated into the ACC/AHA 2005 Guidelines for the Diagnosis and Management of Heart Failure in Adults: a report of the American College of Cardiology Foundation/ American Heart Association Task Force on Practice Guidelines Developed in Collaboration with the International Society for Heart and Lung Transplantation. J Am Coll Cardiol. 2009;53:e1-e90.

23. Gold MR, Thébault C, Linde C, et al. Effect of QRS duration and morphology on cardiac resynchronization therapy outcomes in mild heart failure: results from the Resynchronization Reverses Remodeling in Systolic Left Ventricular Dysfunction (REVERSE) study. Circulation. 2012;126:822-829.

24. Birnie DH, Ha A, Higginson L, et al. Impact of QRS morphology and duration on outcomes after cardiac resynchronization therapy: results from the Resynchronization-Defibrillation for Ambulatory Heart Failure Trial (RAFT). Circ Heart Fail. 2013;6:1190-1198.

25. Sipahi I, Chou JC, Hyden M, Rowland DY, Simon DI, Fang JC. Effect of QRS morphology on clinical event reduction with cardiac resynchronization therapy: meta-analysis of randomized controlled trials. Am Heart J. 2012;163:260-267.e3.

26. Bilchick KC, Kamath S, DiMarco JP, Stukenborg GJ. Bundle-branch block morphology and other predictors of outcome after cardiac resynchronization therapy in Medicare patients. Circulation. 2010;122:2022-2030.

27. Ketchum ES, Jacobson AF, Caldwell JH, et al. Selective improvement in Seattle Heart Failure Model risk stratification using iodine-123 meta-iodobenzylguanidine imaging. J Nucl Cardiol. 2012;19:1007-1016.

28. Barr CS, Lang CC, Hanson J, Arnott M, Kennedy N, Struthers AD. Effects of adding spironolactone to an angiotensin-converting enzyme inhibitor in chronic congestive heart failure secondary to coronary artery disease. Am J Cardiol. 1995;76:1259-1265.

29. Somsen GA, van Vlies B, de Milliano PA, et al. Increased myocardial $\left[{ }^{123} \mathrm{I}\right]-$ metaiodobenzylguanidine uptake after enalapril treatment in patients with chronic heart failure. Heart. 1996;76:218-222.

30. Fukuoka S, Hayashida K, Hirose Y, et al. Use of iodine-123 metaiodobenzylguanidine myocardial imaging to predict the effectiveness of beta-blocker therapy in patients with dilated cardiomyopathy. Eur J Nucl Med. 1997;24:523529.

31. Takeishi Y, Atsumi H, Fujiwara S, Takahashi K, Tomoike H. ACE inhibition reduces cardiac iodine-123-MIBG release in heart failure. J Nucl Med. 1997;38: 1085-1089.

32. Soeki T, Tamura Y, Bandou K, et al. Long-term effects of the angiotensin-converting enzyme inhibitor enalapril on chronic heart failure: examination by ${ }^{123} \mathrm{I}-\mathrm{MIBG}$ imaging. Jpn Heart J. 1998;39:743-751.

33. Toyama T, Aihara Y, Iwasaki T, et al. Cardiac sympathetic activity estimated by ${ }^{123}$ I-MIBG myocardial imaging in patients with dilated cardiomyopathy after $\beta$-blocker or angiotensin-converting enzyme inhibitor therapy. J Nucl Med. 1999;40:217-223.

34. Agostini D, Belin A, Amar MH, et al. Improvement of cardiac neuronal function after carvedilol treatment in dilated cardiomyopathy: a ${ }^{123}$ I-MIBG scintigraphic study. J Nucl Med. 2000;41:845-851.

35. Kasama S, Toyama T, Kumakura H, et al. Effect of spironolactone on cardiac sympathetic nerve activity and left ventricular remodeling in patients with dilated cardiomyopathy. J Am Coll Cardiol. 2003;41:574-581.

36. Toyama T, Hoshizaki H, Seki R, et al. Efficacy of amiodarone treatment on cardiac symptom, function, and sympathetic nerve activity in patients with dilated cardiomyopathy: comparison with beta-blocker therapy. J Nucl Cardiol. 2004;11:134-141.

37. Kasama S, Toyama T, Kumakura H, et al. Effects of candesartan on cardiac sympathetic nerve activity in patients with congestive heart failure and preserved left ventricular ejection fraction. J Am Coll Cardiol. 2005;45:661-667.

38. Suwa M, Otake Y, Moriguchi A, et al. Iodine-123 metaiodobenzylguanidine myocardial scintigraphy for prediction of response to beta-blocker therapy in patients with dilated cardiomyopathy. Am Heart J. 1997;133: 353-358. 